\title{
Multivariate Statistical Analysis Strategies of EELS Spectral Images
}

\author{
Mark H. Van Benthem, Paul G. Kotula, and Ping Lu
}

Sandia National Laboratories, PO Box 5800, Albuquerque, NM 87185 paul.kotula@sandia.gov

Electron energy loss spectroscopy (EELS) holds great promise for routine light-element analysis as well as characterization of localized bonding [1]. When combined with spatial information, as in EELS spectral imaging (SI) [2], it can become even more powerful. The challenge, however, lies in developing robust and effective strategies for statistically analyzing what can be hundreds to tens of thousands of EEL-spectra. Complicating factors in the statistical analysis of EELS-SI data (not present in X-ray data for example [3]) include a moving energy axis due for example to high-voltage instability or electron optics, noise, non-linear signal response with increasing specimen thickness, etc. In this work we describe progress towards the robust analysis of such imperfect EELS-SI data from a simple test specimen.

A $20 \mathrm{~nm} \mathrm{NiO}$ thin film test specimen was deposited by pulsed-laser ablation onto single-crystal $\mathrm{NaCl}$ and then floated off in water and captured on a $\mathrm{Cu}$ grid. In places, the film folded back on itself resulting in a $40 \mathrm{~nm}$ effective thickness NiO film. An EELS-SI consisting of 20 by 20 pixels by 1024 energy-channels covering the spectral region around the oxygen K-edge ( $445 \mathrm{eV}$ to $752 \mathrm{eV}$ with $0.3 \mathrm{eV}$ dispersion) was acquired from a region including $20 \mathrm{~nm}$ and $40 \mathrm{~nm}$ thick $\mathrm{NiO}$. The data were acquired with FEI Company Tecnai F30-ST operated at 300kV in STEM mode and equipped with a Gatan GIF-2001 CCD-based EEL detector. The raw data are shown in Fig 1. On a spectrum by spectrum basis then a background is calculated based upon the logarithm background model [1] of $\ln I(E)=-r \ln E+\ln A$ where $E$ is the energy loss and $A$ and $r$ are fitting parameters. The data (background and non-background) are scaled for Poisson statistics in spectral and spatial domains $[4,5]$ and then the background is subtracted. Then principal components analysis is performed on the scaled background subtracted data resulting in a small number of components. The principal components are then unscaled and the de-noised data are reconstructed from the first three PC's and shown in Fig. 2. Fig 3 shows the first principal component which effectively portrays the two regions in the sample of doubling thickness. Fig 4 is a subsequent principal component which effectively models the shifting energy axis akin to a first derivative. The range of energy shifts modeled in Fig. 4 corresponds to about $2 \mathrm{eV}$. Additional PCs, for the most part, modeled outliers such as X-ray spikes which contaminated the EELS data.

References:

[1] R.F. Egerton, Electron Energy-Loss Spectroscopy in the Electron Microscope, $2^{\text {nd }}$ Ed. (1996) Plenum Press, New York.

[2] C. Jeanguillaume and C. Colliex, Proc. Aussois Workshop (1988) 349.

[3] P.G. Kotula, M.R. Keenan and J.R. Michael. Microsc. Microanal. (2006) 12 36-48.

[4] P.R. Bevington and D.K. Robinson, Data Reduction and Error Analysis for the Physical Sciences, McGraw-Hill Science/Engineering/Math; 3rd edition (2002)

[5] J.R. Taylor, Introduction to Error Analysis, Study of Uncertainties in Physical Measurements, University Science Books; 2nd edition (1997)

Sandia is a multiprogram laboratory operated by Sandia Corporation, a Lockheed Martin Company, for the United Stated Department of Energy's (DOE) National Nuclear Security Administration (NNSA) under contract DE-AC0494AL85000. 

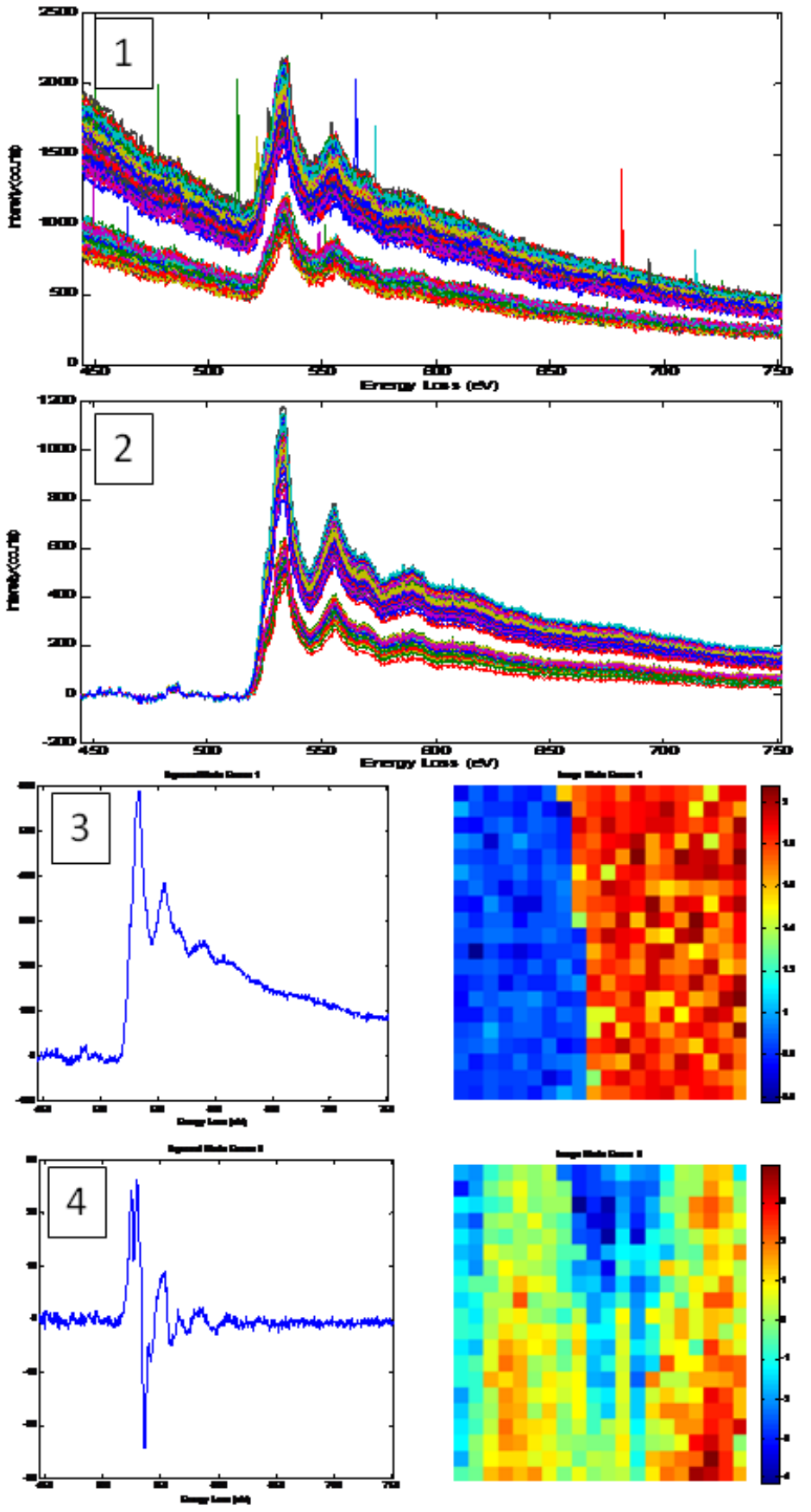

Figure 1. Raw data.

Figure 2. De-noised and background subtracted 3-PC reconstruction of the data.

Figure 3. First principal component spectral shape and corresponding abundance.

Figure 4. Principal component spectral shape and abundance corresponding to the first derivative of the data, which models the non-systematic $2 \mathrm{eV}$ energy-axis shifting due primarily to highvoltage instability. 